\title{
High-Sensitivity Total Reflection X-Ray Fluorescence Spectroscopy of Silicon Wafers Using Synchrotron Radiation ${ }^{\dagger}$
}

\author{
Stephen S. Laderman*1†, Alice Fischer-Colbrie*1, Ayako Shimazaki*2, Kunihiro Miyazaki*2, \\ Sean Brennan ${ }^{* 3}$, Noritaka Takakura*3, Piero PianetTa*3 and Jeffrey B. Kortright*4 \\ ${ }^{*}$ Hewlett-Packard Laboratories, Hewlett-Packard Company, \\ 3500 Deer Creek Road, Palo Alto, CA 94304, USA \\ *2Integrated Circuit Advanced Process Engineering Department, Toshiba Corporation, \\ Komukai Toshiba-cho, Kawasaki 210, Japan \\ ${ }^{*}$ Stanford Synchrotron Radiation Laboratory, Stanford Linear Accelerator Center, \\ P.O. Box 4349, Stanford, CA 94309, USA \\ ${ }^{* 4}$ Center for X-ray Optics, Lawrence Berkeley Laboratory, University of California, \\ Berkeley, CA 94720, USA
}

\begin{abstract}
We explored total reflection X-ray fluorescence spectroscopy (TXRF) with a synchrotron source to achieve high sensitivities to surface metals on silicon wafers. Most recently, we directly demonstrated a sensitivity of $3 \times 10^{8}$ atoms/ $\mathrm{cm}^{2}$ for third-row transition metal elements. The configuration appears to be capable of high sensitivities for a wide range of elements.
\end{abstract}

Keywords Total reflection X-ray fluorescence (TXRF, TRXRF), synchrotron radiation, silicon, VLSI, ULSI, wafer cleaning, microcontamination, synthetic multilayer, multilayer $\mathrm{X}$-ray monochromator

Due to the sensitivity of VLSI circuit yields to low levels of metallic contamination, total reflection X-ray fluorescence (TXRF) analyses based on rotating anode sources are routinely used throughout the semiconductor industry. Nearly every major manufacturer of leadingedge VLSI circuits and many materials and equipment suppliers to these companies have one or more conventional TXRF installations in order to measure trace metal contamination on silicon wafer surfaces. Conventional TXRF equipment is typically reported to have a sensitivity to iron or nickel of nearly $5 \times 10^{9}$ atoms $/ \mathrm{cm}^{2}$. This high sensitivity to impurity contamination, efficient sample handling procedures, mapping and depth profiling capabilities, and the ease of reliably quantifying the data all contributed to the success of the method. However, current advanced wafer surface preparation methods can reproducibly create silicon surfaces that are free of transition metal impurities, such as iron, nickel and copper, to a level below the detection limit of widely available measurement methods, including conventional TXRF. Without more sensitive measurement methods, it is difficult to maintain, evaluate, and improve the processing technology. The motivation for the work

\footnotetext{
$\dagger$ Presented at the 5th Worksop on Total Reflection X-Ray Fluorescence Spectroscopy and Related Spectroscopical Methods, October 17-19, 1994, Tsukuba, Japan.

t† To whom correspondence should be addressed.
}

reported here was the need to develop improved wafer surface analysis methods.

Prior synchrotron radiation TXRF studies have demonstrated detection limits close to $10^{10}$ atoms $/ \mathrm{cm}^{2}$ using single crystal monochromators. ${ }^{1-3}$ Here, we demonstrate more than an order-of-magnitude enhancement in sensitivity over conventional equipment and prior synchrotron radiation studies with a configuration based on synthetic multilayer monochromators. ${ }^{4}$ This configuration provides high sensitivities for a much wider range of elements than is possible when using a rotating anode source. We also describe some steps to be taken to achieve further improvements in its sensitivity and usefulness. As part of this program of study, a detailed analysis of TXRF background signals is underway. Progress along this path has been reported by Takaura $e t$ al., elsewhere. ${ }^{5}$

\section{Sample Preparation and TXRF Configurations}

Two types of samples were studied. The first type was calibration standards used to determine the sensitivities and to calibrate the intensity levels. These standards were made at Toshiba Corporation using well-developed dip contamination methods based on acidic solutions of transition metal salts. ${ }^{6}$ The lateral uniformity and fine contaminant dispersion in these samples were verified by 
conventional TXRF mapping and synchrotron radiation TXRF angle scans. The concentrations were determined with calibrated conventional TXRF and wet chemical methods. ${ }^{6}$ The excellent lateral uniformity made it possible to compare conventional and synchrotron radiation data directly without any geometric correction. The fine dispersion of the contaminants insured that the samples were directly relevant to practical cases of highest interest. The second type of sample was nominally clean samples obtained from wafer vendors, Toshiba, and the Hewlett-Packard Company.

The conventional TXRF data reported here were acquired from Toshiba Corporation and HewlettPackard Company using a Rigaku 3726 TRXRF with a W anode.

The synchrotron radiation TXRF data were collected at the Stanford Synchrotron Radiation Laboratory using a 15 period, $1.4 \mathrm{~T}$ permanent magnet wiggler source. The X-ray beam was focused horizontally and vertically using a toroidal mirror. It was monochromated by reflection from two sequential, identical synthetic multilayers in a parallel geometry. The primary X-ray energy emerging from the multilayer assembly was easily tuned. Here, primary energies of between $10 \mathrm{keV}$ and $11 \mathrm{keV}$ were explored. At a storage ring energy of 3 $\mathrm{GeV}$ and a storage ring current of $100 \mathrm{~mA}$, approximately $10^{13}$ photons/s entered the experimental chamber in a beam close to $2 \mathrm{~mm}$ high and $2 \mathrm{~mm}$ wide. Further details concerning the monochromator and source characteristics have been published elsewhere. ${ }^{7}$ In accord with the sample and detector geometry described next, only the central, brightest portion of this beam contributed to the measured signals.

In the work reported here, the samples were lightly held against a flat chuck by Teflon prongs at their perimeter. Data were collected under vacuum. The wafer surface normal was horizontal, parallel to the dominant polarization direction of the incident X-ray beam. The detector was a single element $\mathrm{Si}(\mathrm{Li})$ solid state detector with a thin aluminized boron nitride window. Outside this window was an additional $4 \mu \mathrm{m}$ thick ultrapure Be window supported in an $\mathrm{Ag}$ ring attached to a W collimator. In addition, a $25 \mu \mathrm{m}$ thick Teflon filter was placed in front of the beryllium window to suppress the Si fluorescence signal. The detector assembly was held close to the wafer surface and the detector's surface normal was parallel to the wafer surface normal. The detector viewed a circle having a diameter close to half a centimeter at the wafer surface. Thus, a $10 \mathrm{~mm}^{2}$ nearly rectangular area centered at the wafer center was examined. A schematic of the sample chamber has been published. ${ }^{8}$

The incident angle was calibrated for each sample by studying the Si fluorescence signal as a function of the Xray incident angle below, close to, and above the critical angle for total reflection. Such scans also demonstrated that the contaminants on the standard samples were not significantly particle-like, but had a very narrow distribution in height, consistent with atomically disperse
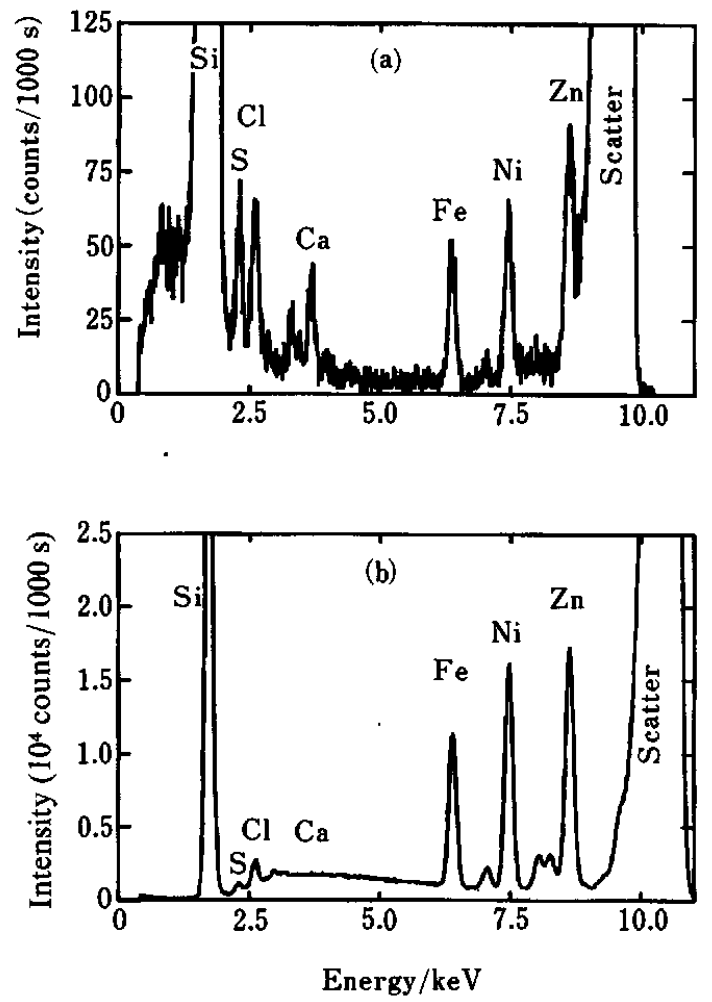

Fig. 1 Conventional (a) and synchrotron radiation (b) TXRF spectra for a sample with $10^{11}$ atoms $/ \mathrm{cm}^{2} \mathrm{Fe}, \mathrm{Ni}$ and $\mathrm{Zn}$.

contamination. In general, impurity fluorescence data were acquired at an angle a few hundredths of a degree below the critical angle.

\section{Experimental Data}

Figure 1 shows the TXRF data obtained from a wafer having $1 \times 10^{11}$ atoms $/ \mathrm{cm}^{2}$ of $\mathrm{Fe}, \mathrm{Ni}$ and $\mathrm{Zn}$ added as intentional contaminants to the surface. The top portion of the figure shows data collected using a rotating anode source. The bottom portion shows data from the same wafer obtained using a synchrotron source. The data in the bottom portion appear to be smoother because the signal-to-noise ratio is higher when the synchrotron source is used. The factor of 200 difference in the ordinate scale reflects this large difference in useful intensity. Using a $3 \sigma$ rule and assuming that the background level is given by the signal levels next to the fluorescence peak, the detection limit for $\mathrm{Ni}$ derived from the conventional spectrum is $5 \times 10^{9}$ atoms $/ \mathrm{cm}^{2}$. For the case of the synchrotron, the detection limit so derived is $3 \times 10^{8}$ atoms $/ \mathrm{cm}^{2}$. This improvement is commensurate with the increased signal level.

Another difference between the conventional and synchrotron radiation data shown in Fig. 1 is the lower background near to the $\mathrm{Zn}$ peak in the case of the synchrotron radiation source. This is a simple example of a general capability made possible by the broadband 


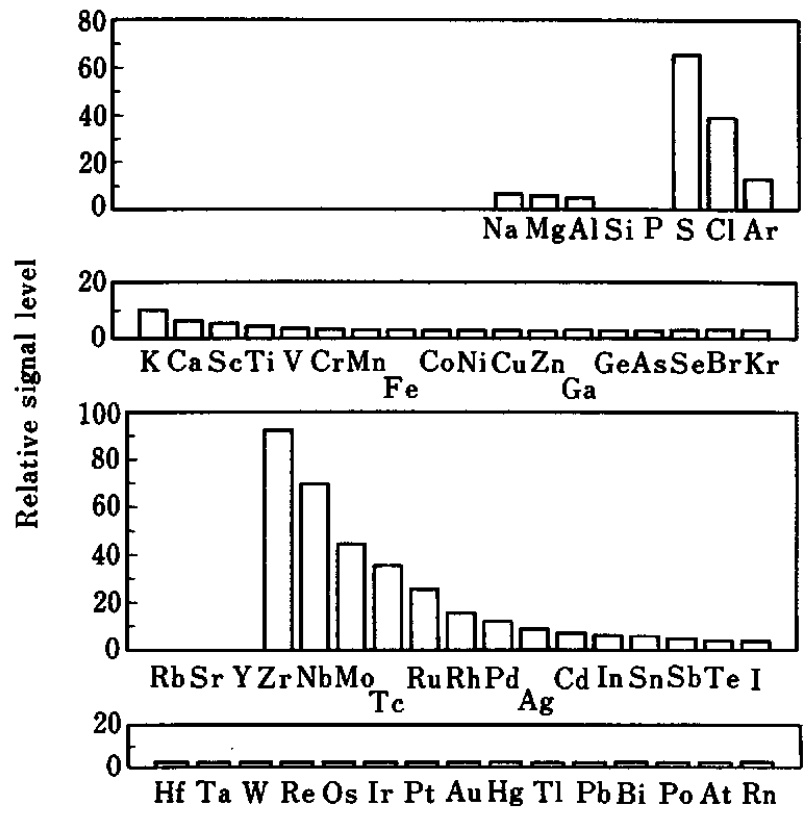

Fig. 2 Calculated synchrotron radiation TXRF sensitivities based on the experimental result for $\mathrm{Ni}$ and the signal rates deduced from atomic parameters.

nature of the synchrotron source and the tunability of the multilayer monochromator. The conventional source has high intensity only near to its anode's fluorescence lines. The line used in the spectrum shown here is so close to the $\mathrm{Zn}$ fluorescence line as to contribute a high background. The synchrotron source, however, has a high intensity over a broad continuum of energies. It is straightforward and convenient to tune the incident $\mathrm{X}$ rays close to an energy which gives high $\mathrm{Zn}$ signal rates, yet low background rates near to the $\mathrm{Zn}$ peak.

In principal, this broadband nature of the synchrotron source and the easily tunable monochromator permit a high sensitivity study of many different impurity elements. A simple calculation was used to provide a first estimate of the sensitivities expected with the configuration used here. Using the ratio of the absorption cross sections, fluorescence yields, and transmission of fluorescence X-rays through the Teflon filter, various elements were compared to the case of $\mathrm{Ni}$. The ratio of the signal to background was assumed to be constant. Although this is not accurate, the result is still useful. The cutoff for the incident X-ray energy was taken to be $15 \mathrm{keV}$, consistent with current multilayer technology. Then, K-absorption edges through the element $\mathrm{Kr}$ and Labsorption edges for the elements $\mathrm{Zr}$ through $\mathrm{Rn}$ could be studied. For elements lighter than Si, the Teflon filter could be removed, since Si $\mathbf{K}_{\alpha}$ fluorescence would no longer be excited. In each case, an optimal incident energy close to the absorption edge was assumed. The resulting estimates are shown in Fig. 2. It can be seen that high signal rates could be obtained for most elements. This configuration is therefore a promising means for studying very many different contaminant

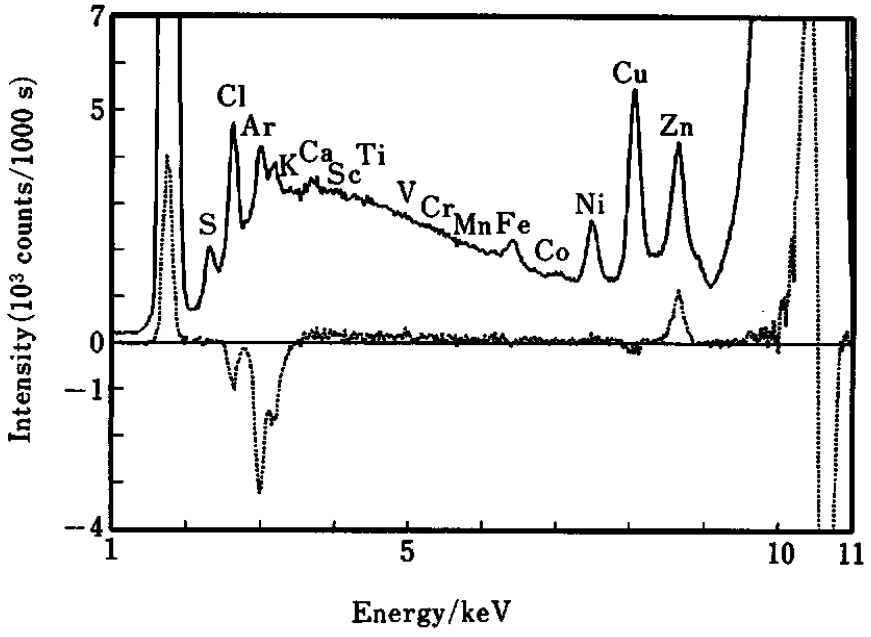

Fig. 3 Synchrotron radiation spectrum from a wafer processed through wet chemical baths associated with an RCA clean plus HF (solid) and a difference spectrum derived by a comparison to the spectrum from a wafer processed without HF (dotted).

elements.

A third difference between the spectra shown in Fig. 1 is the appearance of background near to $2.5 \mathrm{keV}$. The trend in the synchrotron radiation case is due to absorption in the Teflon filter. The smooth background throughout the middle region of the spectrum is due to photoelectron bremsstrahlung. ${ }^{5}$ The bremsstrahlung $\mathrm{X}$-rays are strongly absorbed at lower energies by the $25 \mu \mathrm{m}$ thick Teflon filter.

A repeated study of nominally clean wafers from different sources revealed that peaks always appeared in the vicinity of $\mathrm{Fe}, \mathrm{Ni}, \mathrm{Cu}$ and $\mathrm{Zn}$. By successively placing thick Teflon filters at strategic positions in the apparatus it was determined that the detector assembly, itself, was the source of the peaks. The detector is being redesigned for this application in order to remove these parasitic fluorescence peaks. In the meantime, the analysis has been improved by quantitatively accounting for these background peaks.

For concentrations of impurity atoms as low as those studied here, the $\mathrm{X}$-rays entering the detector assembly are dominated by X-rays scattered by the silicon wafer. Therefore, the parasitic peaks just described were found to be proportional to the intensity of the X-rays scattered from the wafer. In such a case, the cleanliness of two different wafers could sometimes be directly compared by scaling the data sets to the scatter peak near to the incident X-ray energy and subtracting. An example of such a comparison is shown in Fig. 3. Those data come from wafers processed through baths associated with an RCA clean. ${ }^{9}$ One wafer was processed in HF as well. Such single wafer studies should not be considered to be statistically significant. Figure 3 does show that these particular wafers were free of such metals as $\mathrm{V}, \mathrm{Cr}$, and Mn to a very high precision. The difference data show 
a difference in $\mathrm{Zn}$ of about $3 \times 10^{9}$ atoms $/ \mathrm{cm}^{2}$. Using a similar methodology, we also observed differences in the cleanliness of various wafers received from substrate vendors. ${ }^{10}$

\section{Future Prospects}

The data described here show that synchrotron radiation sources make possible higher sensitivities to impurities on Si wafer surfaces than obtained with current conventional TXRF equipment. This improvement offers significant practical advantages to the semiconductor industry. With multiple detector arrays and faster detectors, the configuration could be improved so as to achieve even higher sensitivities.

A limited number of practical applications of the capability have been and will continue to be performed. However, widespread and routine practical use of synchrotron radiation TXRF requires further improvements in detectors, greater access to synchrotron radiation, and state-of-the-art experimental station installations. These changes appear to be straightforward. With them, synchrotron radiation would bring TXRF analysis into a new and useful regime having important applications in the semiconductor industry.

This research was performed, in part, at the Stanford Synchrotron Radiation Laboratory (SSRL), which is operated by the Department of Energy, Office of Basic Energy Sciences. Partial financial support for the synchrotron radiation TXRF sample chamber was provided by the Intel Corporation. The solid state detector system used at SSRL was provided by Fisons Corporation. We acknowledge useful discussions with D. Wherry and experimental support from W. Tompkins and R. Smith.

\section{References}

1. A. Iida, "Advances in $X$-ray Analysis", ed. C. S. Barett, Vol. 35, p. 795, Plenum, New York, 1992.

2. M. C. Madden, D. C. Wherry, P. Pianetta and S. Brennan, Proc. Mater. Res. Soc., 307, 125 (1993).

3. S. S. Laderman and P. Pianetta, Proceedings of the Workshop of Applications of Synchrotron Radiation to Trace Impurity Analysis for Advanced Silicon Processing, Stanford Linear Accelerator Center Technical Report (1992).

4. S. S. Laderman, Bull. Am. Phys. Soc., 39, 514 (1994).

5. N. Takaura, S. Brennan, P. Pianetta, S. S. Laderman, A. Fischer-Colbrie, J. B. Kortright, D. C. Wherry, K. Miyazaki and A. Shimazaki, Adv. X-Ray Chem. Anal. Jpn., 26s, 113 (1995).

6. A. Shimazaki, Proceedings of the Electrochemical Society Symposium on Defects in Silicon II, p. 47, 1991.

7. P. Pianetta, N. Takaura, S. Brennan, W. Tompkins, S. S. Laderman, A. Fischer-Colbrie, A. Shimazaki, K. Miyazaki, M. Madden, D. C. Wherry and J. B. Kortright, Rev. Sci. Instrum., 66, 1293 (1995).

8. S. Brennan, W. Tompkins, N. Takaura, P. Pianetta, S. S. Laderman, A. Fischer-Colbrie, J. B. Kortright, M. C. Madden and D. C. Wherry, Nucl. Instrum. Methods, A347, 417 (1994).

9. W. Kern and D. Puotinen, RCA Rev., 31, 187 (1970).

10. A. Fischer-Colbrie, S. S. Laderman, S. Brennan, N. Takaura, P. Pianetta, A. Shimazaki, K. Miyazaki, J. Kortright and D. C. Wherry, Proceedings of the Second International Symposium on Ultra-clean Processing of Silicon Surfaces, ed. M. Heyns, p. 57, Acco, Leuven/ Amersfoort, 1994.

(Received March 2, 1995) (Accepted April 26, 1995) 